\title{
Marijuana use and risk of prediabetes and diabetes by middle adulthood: the Coronary Artery Risk Development in Young Adults (CARDIA) study
}

\author{
Michael P. Bancks ${ }^{1}$ - Mark J. Pletcher ${ }^{2}$ - Stefan G. Kertesz ${ }^{3,4} \cdot$ Stephen Sidney $^{5}$ • \\ Jamal S. Rana ${ }^{5,6,7}$ • Pamela J. Schreiner ${ }^{1}$
}

Received: 19 April 2015 / Accepted: 6 August 2015 / Published online: 13 September 2015

(C) Springer-Verlag Berlin Heidelberg 2015

\begin{abstract}
Aims/hypothesis The impact of marijuana use on metabolic health is largely unknown. This study sought to clarify the cross-sectional and longitudinal associations between selfreported marijuana use, and prediabetes (defined as fasting glucose 5.6-6.9 mmol/1, $2 \mathrm{~h}$ glucose post OGTT $7.8-11.0 \mathrm{mmol} / \mathrm{l}$ or $\mathrm{HbA}_{1 \mathrm{c}} 5.7-6.4 \%$ [39-47 $\left.\mathrm{mmol} / \mathrm{mol}\right]$ ) and diabetes.

Methods Data from the community-based Coronary Artery Risk Development in Young Adults (CARDIA) study were used to determine marijuana use and the presence of
\end{abstract}

Electronic supplementary material The online version of this article (doi:10.1007/s00125-015-3740-3) contains peer-reviewed but unedited supplementary material, which is available to authorised users.

Michael P. Bancks

banck005@umn.edu

1 Department of Epidemiology and Community Health, School of Public Health, University of Minnesota, 1300 South Second Street, Suite 300, Minneapolis, MN 55454, USA

2 Departments of Epidemiology and Biostatistics and Medicine, School of Medicine, University of California San Francisco, San Francisco, CA, USA

3 Birmingham VA Medical Center, Birmingham, AL, USA

4 Division of Preventive Medicine, School of Medicine, University of Alabama at Birmingham, Birmingham, AL, USA

5 Division of Research, Kaiser Permanente Northern California, Oakland, CA, USA

6 Department of Cardiology, Kaiser Permanente Northern California, Oakland, CA, USA

7 Department of Medicine, University of California San Francisco, San Francisco, CA, USA prediabetes and diabetes among participants. The association between marijuana use and the prevalence of prediabetes and diabetes was examined in 3,034 participants at CARDIA examination year 25 (2010-2011), while the incidence of prediabetes and diabetes according to previous marijuana use was assessed in 3,151 individuals who were free from prediabetes/ diabetes at year 7 (1992-1993) and who returned for at least one of the four subsequent follow-up examinations over 18 years.

Results The percentage of individuals who self-reported current use of marijuana declined over the course of the study's follow-up. After multivariable adjustment, higher odds of prediabetes were found for individuals who reported current use of marijuana (OR 1.65 [95\% CI 1.15, 2.38]) and a lifetime use of 100 times or more (OR 1.49 [95\% CI 1.06, 2.11]), compared with individuals who reported never using marijuana. There was no association between marijuana use and diabetes at CARDIA examination year 25. Over 18 years of follow-up, a greater risk of prediabetes (but not diabetes) was found for individuals who reported a lifetime use of marijuana of 100 times or more (HR 1.39 [95\% CI 1.13, 1.71]), compared with individuals who had never used marijuana.

Conclusions/interpretation Marijuana use in young adulthood is associated with an increased risk of prediabetes by middle adulthood, but not with the development of diabetes by this age.

Keywords Diabetes · Incidence $\cdot$ Longitudinal · Marijuana use · Prediabetes

$\begin{array}{ll}\text { Abbreviations } & \\ \text { CARDIA } & \begin{array}{l}\text { Coronary Artery Risk Development in } \\ \text { Young Adults }\end{array} \\ \text { CRP } & \text { C-reactive protein }\end{array}$




$\begin{array}{ll}\text { EU } & \text { Exercise units } \\ \text { NHANES } & \begin{array}{l}\text { National Health and Nutrition } \\ \text { Examination Survey }\end{array} \\ \text { WC } & \text { Waist circumference } \\ \text { Y0, Y2, .. Y25 } & \text { Year 0, year 2, ... year 25 }\end{array}$

\section{Introduction}

Marijuana is the most frequently used illicit drug in America, with an estimated 18.9 million people aged 12 years or older identifying as current users in 2012 [1]. The prevalence of marijuana use has increased since 2002 [1], and this trend can be expected to continue as states enact policies to permit medicinal or recreational use [2]. Despite the growing movement to legalise marijuana, however, little is known about its effect on metabolic health.

Research on the association between marijuana use and various metabolic indices suggests a paradox in which greater marijuana use is associated with increased caloric consumption, but with decreases in the levels of various metabolic risk indices, including BMI, waist circumference (WC), fasting insulin and HOMA-IR [3-7]. A recent meta-analysis of eight studies suggested that current cannabis smoking is associated with $30 \%$ lower odds of diabetes [8]. However, previous studies have been limited to cross-sectional analyses and might have lacked proper adjustment for confounding. A prospective assessment of health outcomes in relation to prior marijuana use would limit the potential bias that might result from individuals' decisions to alter marijuana exposure based on their own health status.

The purpose of this study was multifaceted. First, we aimed to assess the association between self-reported marijuana use and prediabetes (defined as fasting glucose 5.6-6.9 mmol/l, $2 \mathrm{~h}$ glucose post OGTT $7.8-11.0 \mathrm{mmol} / \mathrm{l}$ or $\mathrm{HbA}_{1 \mathrm{c}} 5.7-6.4 \%$ [39-47 mmol/mol]; see Methods) and diabetes mellitus using cross-sectional and prospective analyses, considering both status (i.e. never, former or current use) and quantity used. Second, we aimed to examine the role of BMI and WC as potential confounding or mediating factors of these associations. Finally, given the varying diabetes risk profiles by race and sex, we sought to evaluate the heterogeneity of effects in the associations by race and sex.

\section{Methods}

Study population The Coronary Artery Risk Development in Young Adults (CARDIA) study is a longitudinal observational study intended to investigate the development of coronary artery disease risk factors in four healthy metropolitan populations (Birmingham, AL; Chicago, IL; Minneapolis, MN; and Oakland, CA, USA) of black and white adults aged
18-30 years at recruitment. Participants were contacted by telephone annually and invited to participate in follow-up examinations at 2, 5, 7, 10, 15, 20 and 25 years after enrolment. Demographic information was obtained, BP and chemistries were measured, and anthropometry and structured questionnaires on manifold health characteristics were conducted, following standardised protocols that were harmonised over time. The institutional review board at each study site granted approval, and informed consent was obtained from the 5,115 participants at enrolment in 1985-1986 (year [Y]0) and at each follow-up examination. Details of the study design have been published previously [9].

Questionnaire, laboratory and physiological measures Participants were asked to fast from all food and beverages (except water) for $12 \mathrm{~h}$ prior to the exam. They were also asked to abstain from any vigorous exercise on the day of the exam and to avoid tobacco use for at least $2 \mathrm{~h}$ prior to the exam. Appointments were scheduled to begin between 07:00 hours and 10:00 hours. Because of diurnal variation in glucose, fasting values were generally drawn by 10:00 hours and oral glucose tolerance testing was not performed if phlebotomy could not be started by 10:30 hours. At each clinical examination, individuals were asked to update their sociodemographic information and were questioned about their medical and family history and individual lifestyle characteristics, including educational attainment, tobacco use (status and frequency), regular alcohol consumption, and moderate and strenuous physical activity. A valid and reliable metric for leisure-time physical activity (exercise units; EU) was developed by CARDIA researchers, as previously described [10].

Venous blood was drawn and serum separation was performed, following which aliquots were stored at $-70^{\circ} \mathrm{C}$ and shipped on dry ice to a central laboratory. Serum glucose was measured using the hexokinase method, and per cent $\mathrm{HbA}_{1 \mathrm{c}}$ was assessed using Tosoh G7 HPLC (Tosoh, San Francisco, CA, USA) and standardised across examinations. The $2 \mathrm{~h}$ serum glucose levels were measured from a standard $2 \mathrm{~h}$ OGTT at Y10, Y20 and Y25. Procedures for collection, storage and determination of plasma lipids and $\mathrm{C}$-reactive protein (CRP) have been previously described [11, 12].

$\mathrm{BP}$ was measured during the resting state, in triplicate with 1 min intermissions, using a random-zero sphygmomanometer at Y0-15 with the first- and fifth-phase Korotkoff sounds corresponding to systolic and diastolic BP, respectively, with the average of the last two measurements used. BP at Y20 and Y25 was measured with an Omron HEM907XL oscillometer (Omron, Schaumburg, IL, USA) and calibrated to the randomzero readings.

Body weight was measured using a calibrated balancebeam scale to the nearest $0.2 \mathrm{~kg}$, with participants in light clothing. Height (without shoes) was measured to the nearest $0.5 \mathrm{~cm}$ using a vertical ruler, with BMI calculated as the 
weight in kilograms divided by the squared height in metres. WC was measured midway between the iliac crest and the lowest lateral portion of the rib cage. Diet was assessed using an interviewer-administered CARDIA diet questionnaire at examination Y0, Y7 and Y20 and a diet score was created, as previously described $[13,14]$. The diet score is a validated measure of dietary quality and a predictor of metabolic health $[15,16]$.

Marijuana and other illicit drug use Participants were asked to complete a detailed, self-administered questionnaire on previous and current use of illicit drugs at each examination; questionnaires are open to the public (www.cardia.dopm.uab.edu/ images/more/pdf/Year25/CARDIA/Form17.pdf) and questions were akin to those found in the National Survey on Drug Use and Health [1]. At each examination, individuals who reported ever using an indicated illicit drug were asked about the number of days of use in the previous 30 days and about lifetime use (i.e. 1-2, 3-9, 10-99 or $\geq 100$ times). The non-medical drugs appraised with this questionnaire included marijuana, crack cocaine or other cocaine, amfetamines and opiates.

Prediabetes and diabetes assessment Individuals were identified as having prediabetes or diabetes according to ADA glycaemic cut-off points. The ADA defines prediabetes as fasting glucose 5.6-6.9 mmol/1, $2 \mathrm{~h}$ glucose post OGTT 7.8$11.0 \mathrm{mmol} / \mathrm{l}$ or $\mathrm{HbA}_{1 \mathrm{c}} 5.7-6.4 \%$ (39-47 mmol$/ \mathrm{mol}$ ) [17]. The cut-off points for diabetes include fasting glucose $\geq 7.0 \mathrm{mmol} / \mathrm{l}$, $2 \mathrm{~h}$ glucose post OGTT $\geq 11.1 \mathrm{mmol} / 1$ or $\mathrm{HbA}_{1 \mathrm{c}} \geq 6.5 \%$ ( $\geq 48 \mathrm{mmol} / \mathrm{mol}$ ) [17]. Individuals who reported the use of diabetes medications were also identified as diabetes cases.

Population for analysis We used two distinct methods to evaluate the metabolic effects of self-reported marijuana use in CARDIA participants. First, to assess the cross-sectional association between self-reported marijuana use and prediabetes and diabetes, data from examination Y25 were analysed. For this cross-sectional evaluation, of the 3,496 individuals present at examination Y25, we excluded those who had fasted for less than $8 \mathrm{~h}$ prior to the visit $(n=209)$ and those with an undeterminable diabetes status $(n=1)$ or missing relevant covariate information $(n=252)$, resulting in a sample of 3,034 participants. Individuals who had diabetes at Y25 $(n=357)$ were excluded from prediabetes analyses (to mitigate selection bias), and prediabetes status was undetermined for one individual. Therefore, 2,676 individuals were included in prediabetes analyses.

The second analytical approach was to prospectively evaluate the association between self-reported marijuana use and incident prediabetes and diabetes. Fasting glucose was not measured at CARDIA Y2 and Y5, and diabetes status was determined by medication use. In order to include the ADA criteria in determining incident diabetes at each examination, data from examination $\mathrm{Y} 7$ were used as the analysis baseline. Individuals were excluded from analysis if they did not participate in the Y7 examination $(n=1,029)$, presented with a fasting time of less than $8 \mathrm{~h}$ prior to the $\mathrm{Y} 7$ examination $(n=403)$, did not return for follow-up in all of Y10-25 $(n=162)$ or were missing covariate information at the Y7 examination $(n=268)$. When assessing marijuana use and incident diabetes, individuals with prevalent diabetes at Y7 or those whose diabetes status was undetermined on follow-up were excluded $(n=102)$, resulting in an analysis sample of 3,151 participants.

For the association between marijuana use and incident prediabetes, 495 people were excluded based on baseline prediabetes, diabetes and underdetermined prediabetes status on follow-up, giving a final analysis sample of 2,758 participants. Those excluded were on average older and were more likely to be male, African-American and less educated, with a longer history of smoking, higher levels of fasting glucose and CRP, and greater lifetime frequency of marijuana use compared with the included participants.

Statistical analysis Categories of all unique forms of selfreported drug use were determined by status (i.e. never/former/ current use) and total use (i.e. lifetime frequency). Former use was defined as an affirmative response to the question 'Ever use?', but with no reported use in the previous 30 days. Current use was determined by a report of use on one or more of the last 30 days.

Along with other illicit drug use, we considered several additional covariates as potential confounders. Cigarette smoking status was based entirely on current use. Regular alcohol consumption was classified as none, up to one drink daily and more than one drink daily. Educational attainment was characterised into three groups: $\leq 12$ (high school), 13-16 (any college) or $>16$ years of education ( $>4$ years' college). Systolic BP, BMI, WC, LDL- and HDL-cholesterol, and CRP variables were modelled continuously, as were physical activity and diet scores. Antihypertensive and lipid-lowering medication use was taken into account in models that included adjustment for BP and cholesterol levels. Given the strength of association between BMI and diabetes and to reduce potential residual confounding, all adjusted models containing $\mathrm{BMI}$ also included a $\mathrm{BMI}^{2}$ term to account for a possible nonlinear relationship.

Participant characteristics were calculated across categories of self-reported marijuana use. Univariate models were used to assess the crude direction and magnitude of each association, with sequential models adjusting for the potential confounders noted above. The association between marijuana use and the presence of prediabetes and diabetes at CARDIA examination Y25 was estimated with logistic regression, obtaining crude and adjusted ORs and 95\% CIs. For longitudinal analyses, crude and adjusted HRs and 95\% CIs were 
estimated using Cox proportional hazards models. Contributed person-time to the study was calculated as the duration from date of examination Y7 to either: (1) the examination at which the event of interest (i.e. prediabetes or diabetes) was ascertained; or (2) administrative censoring of the participant's last examination visit. The proportional hazards assumption was assessed by including a product term between marijuana use category and natural log of contributed person-time.

To investigate whether the risk of prediabetes and diabetes according to marijuana use differed by sex or race, separate multiplicative interactions were tested by adding product terms to the proportional hazards model. Sensitivity analyses were also performed, repeating the main analyses with data from different CARDIA examination years to confirm whether associations were similar regardless of the examination from which participant data were used. For example, for cross-sectional analyses, marijuana use and prevalence of prediabetes and diabetes were assessed using data from each CARDIA examination Y0-20. For prospective analyses, we assessed marijuana use at each CARDIA examination Y0-20 and incidence of prediabetes and diabetes through to Y25. Statistical analyses were performed using SAS statistical software version 9.3 (SAS Institute, Cary, NC, USA).

\section{Results}

The self-reported marijuana use status of the individuals present at each examination is displayed in Fig. 1. The per cent of individuals reporting 'never' or 'current' use of marijuana declined over time, while the per cent who reported 'former' use of marijuana increased, particularly in the early years.

Baseline participant characteristics for the prospective analysis are presented in Table 1 by category of lifetime frequency of marijuana use. In both the cross-sectional and longitudinal analyses, older age, male sex, white race, current smoking, greater daily alcohol consumption, current use of

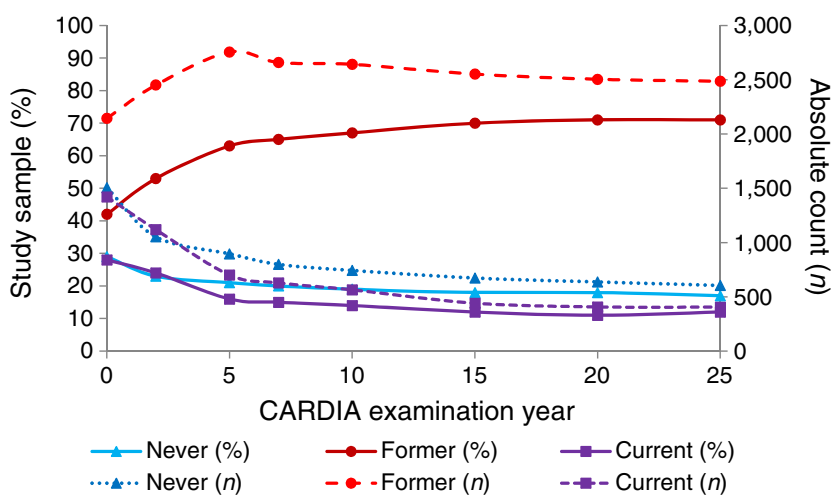

Fig. 1 Self-reported marijuana use status of individuals across the examination years of the CARDIA study marijuana, other illicit drug use and greater participation in physical activity were all associated with a greater lifetime frequency of marijuana use, while longer time in education and greater BMI were associated with lower frequency of marijuana use.

At CARDIA examination Y25, $45 \%$ of the analysis population had prediabetes $(n=1,193)$. Marijuana use was associated with higher odds of prediabetes, regardless of status or frequency of use, before and after adjustment (Table 2). Specifically, individuals who reported current use and those who reported a lifetime use of $\geq 100$ times had significantly higher odds of prediabetes compared with those who reported never using marijuana. The greatest attenuation of estimates was observed with adjustment for age, sex, and race, while the greatest strengthening of estimates was observed when use of other illicit drugs was included.

There were 357 cases of prevalent diabetes identified at Y25 for the cross-sectional analysis. Without adjustment for covariates, individuals who reported a history of marijuana use when marijuana use was modelled by status or lifetime frequency had marginally lower odds of diabetes compared with never-users (Table 2). Adjustment for demographic and lifestyle characteristics reversed the apparent direction of the association from $<1$ to $>1$, although the $95 \%$ CIs continued to span 1 (Table 2). Estimates were most sensitive to adjustment for alcohol use, field centre, BP (or medication use) and use of other illicit drugs. The results for the prediabetes and diabetes analyses did not materially change when CRP level was excluded from the models or when $\mathrm{BMI}, \mathrm{BMI}^{2}$ and $\mathrm{WC}$ were inserted into the models.

More than half of the participants without prediabetes or diabetes at the start of follow-up developed prediabetes over an average of 13.8 years of follow-up $(51 \% ; n=1,410)$. Table 3 presents the crude and fully adjusted HRs with $95 \%$ CIs and crude incidence rates for prediabetes and diabetes according to self-reported marijuana use category. Unadjusted models for the association between marijuana use and incident prediabetes found a suggestive increase in the hazard for prediabetes for individuals with the greatest frequency of use at baseline ( $\geq 100$ times). Adjustment for covariates strengthened the observed association in this group, with the $95 \%$ CIs no longer spanning 1 after adjustment for demographics, tobacco use, alcohol intake and dietary pattern. Compared with those who reported never using marijuana, individuals who reported use of $\geq 100$ times had a significantly increased risk for prediabetes (HR 1.39 [95\% CI 1.13, 1.71]), after adjustment for demographic, lifestyle and clinical characteristics.

There were 351 incident cases of diabetes identified during 50,569 years of follow-up in the prospective analysis, giving an overall crude incidence of 694 cases per 100,000 personyears. In unadjusted analysis, a decreased risk of diabetes was found for those who reported marijuana use compared with never-users, but this did not attain statistical significance. The associations were attenuated after adjustment for basic 
Table 1 Participant characteristics according to self-reported marijuana use at examination Y7 (1992-1993)

\begin{tabular}{|c|c|c|c|c|}
\hline \multirow[t]{2}{*}{ Characteristic } & \multicolumn{4}{|c|}{ Self-reported lifetime marijuana use } \\
\hline & Never $(n=625)$ & $1-9$ times $(n=738)$ & $10-99$ times $(n=774)$ & $\geq 100$ times $(n=1,014)$ \\
\hline Age, years & $31.8 \pm 3.9$ & $32.4 \pm 3.7$ & $32.9 \pm 3.5$ & $33.1 \pm 3.3$ \\
\hline Female, \% & 59.5 & 63.0 & 64.9 & 38.3 \\
\hline White, \% & 45.6 & 53.5 & 60.3 & 59.3 \\
\hline \multicolumn{5}{|l|}{ Highest education, $\%$} \\
\hline High school & 22.7 & 20.9 & 20.3 & 34.1 \\
\hline Any college & 56.2 & 57.3 & 57.0 & 51.0 \\
\hline$>4$ years' college & 21.1 & 21.8 & 22.7 & 14.9 \\
\hline A priori diet score & $57.6 \pm 11.7$ & $61.9 \pm 12.3$ & $64.7 \pm 12.7$ & $64.7 \pm 12.5$ \\
\hline Physical activity, EU & $296.6 \pm 260.7$ & $319.5 \pm 263.7$ & $328.4 \pm 248.8$ & $390.7 \pm 274.0$ \\
\hline BMI, $\mathrm{kg} / \mathrm{m}^{2}$ & $27.4 \pm 6.5$ & $26.8 \pm 6.4$ & $26.1 \pm 6.0$ & $26.4 \pm 5.5$ \\
\hline $\mathrm{WC}, \mathrm{cm}$ & $84.5 \pm 14.6$ & $83.1 \pm 13.7$ & $81.5 \pm 13.8$ & $84.9 \pm 13.1$ \\
\hline Fasting glucose, mmol/1 & $4.9 \pm 0.5$ & $4.9 \pm 0.4$ & $4.9 \pm 0.5$ & $5.0 \pm 0.5$ \\
\hline Systolic BP, mmHg & $109.0 \pm 12.7$ & $107.9 \pm 12.2$ & $106.6 \pm 11.3$ & $109.3 \pm 11.8$ \\
\hline Diastolic BP, mmHg & $70.5 \pm 10.1$ & $69.5 \pm 9.8$ & $67.9 \pm 9.5$ & $69.3 \pm 9.7$ \\
\hline Antihypertension medications, $\%$ & 2.1 & 1.8 & 1.7 & 1.2 \\
\hline Total cholesterol, mmol/1 & $4.5 \pm 0.9$ & $4.6 \pm 0.8$ & $4.5 \pm 0.8$ & $4.6 \pm 0.9$ \\
\hline LDL-cholesterol, mmol/1 & $2.8 \pm 0.8$ & $2.8 \pm 0.8$ & $2.7 \pm 0.8$ & $2.8 \pm 0.8$ \\
\hline HDL-cholesterol, mmol/1 & $1.3 \pm 0.3$ & $1.4 \pm 0.3$ & $1.4 \pm 0.4$ & $1.3 \pm 0.4$ \\
\hline Lipid-lowering medications, $\%$ & 0.2 & 0.1 & 0.0 & 0.3 \\
\hline $\mathrm{CRP}, \mathrm{nmol} / 1$ & $21.0 \pm 30.5$ & $26.7 \pm 40.0$ & $31.4 \pm 40.0$ & $37.1 \pm 45.7$ \\
\hline \multicolumn{5}{|l|}{ Smoking status, $\%$} \\
\hline Never & 89.1 & 76.5 & 51.0 & 35.2 \\
\hline Former & 3.2 & 10.4 & 21.1 & 26.2 \\
\hline Current & 7.7 & 13.1 & 27.9 & 38.6 \\
\hline \multicolumn{5}{|l|}{ Alcohol consumption, $\%$} \\
\hline No daily consumption & 73.7 & 50.5 & 34.6 & 30.3 \\
\hline$\leq 1$ drink/day & 22.1 & 38.8 & 47.2 & 39.2 \\
\hline$>1$ drink/day & 4.2 & 10.7 & 18.2 & 30.6 \\
\hline Current marijuana use, $\%$ & 0.0 & 1.5 & 9.8 & 38.6 \\
\hline \multicolumn{5}{|l|}{ Crack cocaine use, $\%$} \\
\hline Ever use & 1.0 & 12.3 & 45.3 & 74.6 \\
\hline$>10$ times lifetime use & 0.2 & 1.9 & 10.1 & 34.1 \\
\hline \multicolumn{5}{|l|}{ Other cocaine use, $\%$} \\
\hline Ever use & 0.8 & 15.6 & 50.6 & 82.2 \\
\hline$>10$ times lifetime use & 0.0 & 1.9 & 11.2 & 40.2 \\
\hline \multicolumn{5}{|l|}{ Amfetamine use, $\%$} \\
\hline Ever use & 1.6 & 10.6 & 38.3 & 67.5 \\
\hline$>10$ times lifetime use & 0.5 & 1.5 & 9.2 & 29.7 \\
\hline \multicolumn{5}{|l|}{ Heroin use, $\%$} \\
\hline Ever use & 2.1 & 2.4 & 8.3 & 22.2 \\
\hline$>10$ times lifetime use & 0.0 & 0.1 & 1.4 & 5.5 \\
\hline
\end{tabular}

Continuous variables are means $\pm \mathrm{SD}$

Categorical variables are per cent within column

demographic and lifestyle characteristics; further adjustment for dietary pattern and BP resulted in the greatest attenuation of estimates. Irrespective of the outcome (i.e. prediabetes or diabetes), the results did not differ when fasting glucose, BMI 
Table 2 ORs ( $95 \%$ CIs) for the prevalence of prediabetes and diabetes in CARDIA Y25 according to self-reported marijuana use category in Y25

\begin{tabular}{|c|c|c|c|c|c|c|c|c|}
\hline \multirow{2}{*}{$\begin{array}{l}\text { Self- } \\
\text { reported } \\
\text { marijuana } \\
\text { use }\end{array}$} & \multicolumn{4}{|l|}{ Prediabetes } & \multicolumn{4}{|l|}{ Diabetes } \\
\hline & $\begin{array}{l}\text { No. } \\
\text { cases } / n\end{array}$ & Crude OR & Adjusted $\mathrm{OR}^{\mathrm{a}}$ & Adjusted $\mathrm{OR}^{\mathrm{b}}$ & $\begin{array}{l}\text { No. } \\
\text { cases } / n\end{array}$ & Crude OR & Adjusted $\mathrm{OR}^{\mathrm{a}}$ & Adjusted $\mathrm{OR}^{\mathrm{b}}$ \\
\hline Never & $198 / 475$ & 1.00 (ref.) & 1.00 (ref.) & 1.00 (ref.) & $71 / 547$ & 1.00 (ref.) & 1.00 (ref.) & 1.00 (ref.) \\
\hline Former & $833 / 1,895$ & $1.10(0.90,1.35)$ & $1.23(0.96,1.58)$ & $1.24(0.97,1.58)$ & $256 / 2,151$ & $0.91(0.68,1.20)$ & $1.25(0.87,1.79)$ & $1.23(0.85,1.78)$ \\
\hline Current & $162 / 306$ & $1.57(1.18,2.10)$ & $1.65(1.15,2.38)$ & $1.66(1.15,2.38)$ & $30 / 336$ & $0.66(0.42,1.03)$ & $1.18(0.67,2.10)$ & $1.16(0.64,2.08)$ \\
\hline \multicolumn{9}{|l|}{ Lifetime use } \\
\hline 1-9 times & $390 / 897$ & $1.08(0.86,1.35)$ & $1.21(0.94,1.56)$ & $1.21(0.94,1.57)$ & $128 / 1,025$ & $0.96(0.70,1.31)$ & $1.21(0.84,1.76)$ & $1.20(0.82,1.75)$ \\
\hline 10-99 times & $253 / 609$ & $0.99(0.78,1.27)$ & $1.29(0.95,1.77)$ & $1.28(0.94,1.75)$ & $72 / 681$ & $0.79(0.56,1.12)$ & $1.35(0.85,2.16)$ & $1.34(0.83,2.18)$ \\
\hline$\geq 100$ times & $352 / 695$ & $1.44(1.14,1.82)$ & $1.49(1.06,2.11)$ & $1.38(0.96,1.97)$ & $86 / 781$ & $0.83(0.59,1.16)$ & $1.42(0.85,2.38)$ & $1.28(0.75,2.18)$ \\
\hline
\end{tabular}

${ }^{a}$ Models adjusted for age, sex, race, smoking, alcohol, education, field centre, SBP, CRP, physical activity and use of other illicit drugs.

${ }^{\mathrm{b}}$ As for the model described above, with the addition of BMI, $\mathrm{BMI}^{2}$ and $\mathrm{WC}$

and pack-years of cigarette smoking were included in the final model.

For all prospective analyses, inclusion of age and illicit drug use at baseline in the model resulted in considerable strengthening of estimates. Otherwise, any strengthening of the associations with the incremental inclusion of individual variables was far less in magnitude and balanced by the covariates that attenuated the associations. Formal tests of interaction were not significant $(p>0.10)$ for any of the potential effect modifiers for any of the analyses in this study. No violations to the proportional hazards assumption were detected. Results from sensitivity analyses confirmed the primary analyses (results not shown); patterns of the associations were similar and did not depend on the year from which participant data were used.

Electronic supplementary material (ESM) Table 1 shows the fasting glucose levels at the time of censoring: either the examination at which prediabetes and diabetes was ascertained or administrative censoring of the last examination visit. There was no observable linear trend in glucose levels at the time of censoring across marijuana use categories for diabetes. However, a statistically significant positive linear trend

Table 3 HRs (95\% CIs) for incident prediabetes and diabetes by self-reported marijuana use; CARDIA examination Y7-25 (1992-1993 to 2010 2011)

Lifetime frequency of marijuana use

Never use $\quad 1-9$ times $\quad 10-99$ times $\quad \geq 100$ times $\quad p$ for trend ${ }^{\text {a }}$

Prediabetes

\begin{tabular}{|c|c|c|c|c|c|}
\hline No. cases $/ n$ & $275 / 548$ & $329 / 660$ & $333 / 692$ & $473 / 858$ & \\
\hline Incidence, per 100,000 person-years & 3,628 & 3,607 & 3,415 & 4,084 & \\
\hline Crude HR $(95 \% \mathrm{CI})$ & 1.00 (ref.) & $0.99(0.84,1.16)$ & $0.93(0.79,1.09)$ & $1.15(0.99,1.33)$ & 0.07 \\
\hline Adjusted HR $(95 \% \mathrm{CI})^{\mathrm{b}}$ & 1.00 (ref.) & $1.13(0.96,1.34)$ & $1.21(1.00,1.45)$ & $1.39(1.13,1.71)$ & $<0.01$ \\
\hline Adjusted HR $(95 \% \mathrm{CI})^{\mathrm{c}}$ & 1.00 (ref.) & $1.13(0.95,1.33)$ & $1.21(1.00,1.45)$ & $1.40(1.13,1.72)$ & $<0.01$ \\
\hline \multicolumn{6}{|l|}{ etes } \\
\hline No. cases $/ n$ & $81 / 625$ & $79 / 738$ & $85 / 774$ & $106 / 1,014$ & \\
\hline Incidence per 100,000 person-years & 811 & 667 & 685 & 649 & \\
\hline Crude HR $(95 \%$ CI $)$ & 1.00 (ref.) & $0.82(0.60,1.11)$ & $0.84(0.62,1.14)$ & $0.78(0.59,1.05)$ & 0.15 \\
\hline Adjusted HR $(95 \% \text { CI })^{\mathrm{b}}$ & 1.00 (ref.) & $0.94(0.68,1.29)$ & $1.28(0.90,1.83)$ & $1.10(0.74,1.64)$ & 0.45 \\
\hline Adjusted HR $(95 \% \mathrm{CI})^{\mathrm{c}}$ & 1.00 (ref.) & $0.93(0.68,1.29)$ & $1.30(0.91,1.86)$ & $1.16(0.77,1.74)$ & 0.32 \\
\hline
\end{tabular}

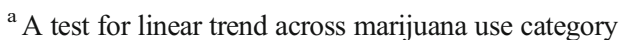

${ }^{\mathrm{b}}$ Adjusted for Y7 covariate values for age, sex, race, smoking, alcohol, education, field centre, diet quality, physical activity, SBP, LDL-cholesterol, HDL-cholesterol, medication use and use of other illicit drugs

${ }^{\mathrm{c}}$ As for the model described above, with the addition of $\mathrm{BMI}, \mathrm{BMI}^{2}$ and $\mathrm{WC}$ 
was observed for prediabetes, although this was no longer apparent after adjustment for illicit drug use.

\section{Discussion}

In this cohort of healthy men and women, marijuana use was associated with a higher prevalence of prediabetes during middle adulthood after controlling for potential confounding variables, but was not associated with the presence of diabetes at this age. Similarly, marijuana use in young adulthood was associated with the incidence of prediabetes in middle age. The greatest lifetime frequency of use ( $\geq 100$ times) at baseline was associated with the highest incidence of prediabetes over the study's followup, compared with participants who reported never using marijuana. Marijuana use was not associated with the incidence of diabetes. Marijuana use was modelled categorically in two different ways (current/former/never, and with reference to lifetime frequency), contributing to the interpretation and robustness of these findings.

The findings of this study are important, given the previously reported associations of marijuana use with various metabolic outcomes [3, 5-7]. The impact of BMI on the association between marijuana use and incident diabetes and prediabetes is unclear (i.e. whether it has no effect, or is a potential confounder or mediator). In this study, the results were unchanged with the addition of BMI, $\mathrm{BMI}^{2}$ and $\mathrm{WC}$ to the statistical model, consistent with the minimal estimate shift observed in a recent meta-analysis [8], and we found no cross-sectional association between marijuana use and BMI (data not shown), in contrast to previous findings on marijuana use and metabolic health [5-8]. A previous study assessed marijuana use in relation to obesity status in two population-based, nationally representative samples of US adults [5]. Using the National Epidemiologic Survey on Alcohol and Related Conditions, researchers found that individuals who reported cannabis use on $\geq 3$ days per week had $39 \%$ lower odds of obesity compared with individuals who reported no use in the past 12 months, after adjustment for demographics, education, marital status, religion and tobacco smoking status. This association was attenuated when researchers studied individuals from the National Comorbidity Survey-Replication; adjusted estimates no longer attained statistical significance (OR 0.73 [95\% CI 0.43, 1.23]) [5]. The prevalence of current marijuana use was $<8 \%$ in this study, and the prevalence of use among young adults was below the national average and that found in our cross-sectional analysis $[1,5]$.

The association between marijuana use and the prevalence of diabetes has also been examined in the National Health and Nutrition Examination Survey (NHANES) III population. The researchers reported that marijuana use was associated with a lower odds of diabetes (OR 0.36 [95\% CI 0.24, 0.55]) after adjustment for manifold demographic, lifestyle and clinical characteristics [6]. The study population was restricted to individuals aged $20-59$ years; excluded $25 \%$ of the potential analysis population because of missing laboratory data; and, when examining age-stratified analyses ( $>40$ and $\leq 40$ years), found the association was restricted to the older age stratum [6]. A recent meta-analysis of eight independent replications from NHANES and the National Surveys on Drug Use and Health found a summary adjusted OR of 0.7 (95\% CI $0.6,0.8)$ for the association of current marijuana use and prevalent diabetes; however, both marijuana use and diabetes status were ascertained via self-report [8]. These associations might result from the self-exclusion of unhealthy individuals who frequently use marijuana from study participation, resulting in an underestimation of diabetes cases in marijuana users, and from reverse causation where individuals with diabetes abstain from marijuana use in older age because of concerns about and awareness of their health status [6, 8].

Recent analysis of NHANES 2005-2010 participants found marijuana use to be associated with lower levels of fasting insulin and HOMA-IR, and a decreased WC compared with individuals who reported never using marijuana, after adjustment for relevant covariates; however, no association was found between current marijuana use and fasting glucose, $\mathrm{HbA}_{1 \mathrm{c}}$ or BMI [7]. In a small study $(n=60)$ of otherwise healthy individuals, after matching cannabis users to nonusers by sex, ethnicity, age and BMI, adipocyte insulin sensitivity was found to be higher in cannabis users compared with non-users; however, skeletal muscle insulin sensitivity, insulin secretion, fasting insulin and glucose, and $\mathrm{HbA}_{1 \mathrm{c}}$ were not different between the two groups [18]. Significant differences in diet quality between the two groups were noted, and the effect of tobacco use on the results is unknown [18].

Previous epidemiological research has cited animal models investigating the potential mechanisms underlying the metabolic effects of marijuana. Potential anti-inflammatory effects and improved metabolism by actions through the cannabinoid receptors have been suggested to reduce the progression of type 1 diabetes, improve beta cell function and decrease weight in mouse models $[19,20]$. However, no models have assessed this association in healthy mice, and these studies administered cannabis/cannabidiol via ingestion or intravenously. The mode of administration and the dose should be considered when extending these results to public health studies, as the most common modes of consuming cannabis among the general population are cigarettes, pipes and bongs, in which the user inhales the chemical compounds in smoke form and the quantity consumed varies from user to user [21]. Given the potential of marijuana smoke to increase the production of reactive oxygen species and oxidative stress, any potential anti-inflammatory benefit might be countered by detrimental oxidative effects from intake by smoking [22]. 
Research on the prospective evaluation of marijuana use on metabolic health is scant. It is unclear how marijuana use could place an individual at increased risk for prediabetes yet not diabetes. This is a potential study limitation, and may reflect a spurious prediabetes association. Similarly, it is possible that it is an artefact arising from our exclusion criteria disproportionately affecting those with higher levels of marijuana use and greater potential for the development of diabetes. Individuals excluded from our analysis generally had higher levels of marijuana use and less favourable levels of traditional diabetes risk factors and were, historically, more likely to develop diabetes. Alternatively, the effect of marijuana use might have a more noticeable impact on glucose metabolism in the prediabetes range compared with the diabetes range, when traditional diabetes risk factors are far less favourable and might dominate over any effect of marijuana. This might explain the differing results in the linear trend of fasting glucose level at censoring.

There are plausible ways to reconcile the seemingly contradictory tendencies between this prospective analysis (where earlier marijuana use predicts later prediabetes), animal and cellular models, and prior cross-sectional findings in which current marijuana use coincided with a lower prevalence of prediabetes and diabetes. We speculate, for example, that some people in ill health might choose to quit marijuana as a result of a physician's recommendation to abstain from tobacco and other substances or a general concern for their health, or because of more complicated associations between poor health, income and drug access. This speculation awaits confirmation. In addition, previous work has not accounted for the use of other illicit drugs [5-8]. While illicit drug use per se might not cause a decline in metabolic health, it might be an indicator of the propensity to use drugs or overall deleterious health behaviour, or cause declines in overall health [23, 24].

Limitations When weighing the contribution of this study to our understanding of the effect of marijuana use on metabolism, certain limitations apply. Foremost, our main exposure variable was determined by self-report. Self-report of marijuana use has been shown to have good validity compared with objective measures, and an objective measure is not typically available for observation periods that span many years [25]. In the CARDIA study, participants were asked about illicit drug use at each examination and the cohort experienced no adverse consequences from reporting, potentially mitigating under-reporting.

Prospective analyses are unlikely to be affected by differential reporting of baseline exposure by case status, particularly in this analysis where outcomes occurred, on average, 14-16 years after the initial response. For the prospective analyses, participant exposure level and other characteristics reflected those measured at CARDIA examination $\mathrm{Y} 7$ and were not updated over time. We found that marijuana use status levelled off between the Y7 and Y10 examinations, which might reflect individuals exiting young adulthood - on average 32 years old, traditionally past peak drug use and established into a habitual frequency of use [1]. Although smoking is the most common way of using marijuana, the mode of marijuana use was not specified in responses. It is unclear if the effect of marijuana differs by mode of use.

Strengths Strengths of this study include the objective measure of prediabetes and diabetes status, ascertainment of manifold confounding factors and marijuana use response rates similar to those noted in young adults in the general US population [26]. We found consistent results on cross-sectional and prospective analyses and when we performed sensitivity analyses, repeating the main analyses using data from different CARDIA examination years.

Conclusion In conclusion, marijuana use, by status or lifetime frequency, was not associated with the incidence or prevalence of diabetes after adjustment for potential confounding factors. However, marijuana use was associated with the development and prevalence of prediabetes after adjustment. Specifically, the occurrence of prediabetes in middle adulthood was significantly more likely among individuals who reported using marijuana more than 100 times by young adulthood. These results contrast with previous findings on marijuana use and metabolic health. Future studies should aim to objectively measure the mode and quantity of marijuana use in relation to prospective metabolic health.

Acknowledgements We thank the participants and staff of the CARDIA study; this work would not have been possible without them.

Funding MPB was supported by the National Institutes of Health (NIH) Training Grant T32HL007779 to conduct the current work. The CARDIA study is conducted and supported by the National Heart, Lung, and Blood Institute (NHLBI) in collaboration with the University of Alabama at Birmingham (HHSN268201300025C and HHSN268201300026C), Northwestern University (HHSN268201300027C), the University of Minnesota (HHSN268201300028C), the Kaiser Foundation Research Institute (HHSN268201300029C) and Johns Hopkins University School of Medicine (HHSN268200900041C). The CARDIA study is also partially supported by the Intramural Research Program of the National Institute on Aging (NIA) and an intra-agency agreement between the NIA and NHLBI (AG0005). This manuscript has been reviewed by CARDIA for scientific content. The content is solely the responsibility of the authors and does not necessarily represent the official views of the NIH.

Duality of interest The authors declare that there is no duality of interest associated with this manuscript.

Author contributions All authors fulfil the contribution requirements for authorship credit, including, for each author listed: (1) substantial contributions to conception and design, acquisition of data, or analysis and interpretation of data; (2) drafting the article or revising it critically for important intellectual content; and (3) final approval of the version to be published. MPB is the guarantor of this work. 


\section{References}

1. Substance Abuse and Mental Health Services Administration (2013) Results from the 2012 National Survey on Drug Use and Health: summary of national findings, NSDUH Series H-46, HHS Publication No. (SMA) 13-4795. Substance Abuse and Mental Health Services Administration, Rockville

2. Leon K, Weitzer R (2014) Legalizing recreational marijuana: comparing ballot outcomes in four states. J Qual Crim Just Criminol 2: 193-218

3. Rodondi N, Pletcher MJ, Liu K, Hulley SB, Sidney S (2006) Marijuana use, diet, body mass index, and cardiovascular risk factors (from the CARDIA study). Am J Cardiol 98:478-484

4. Smit E, Crespo CJ (2001) Dietary intake and nutritional status of US adult marijuana users: results from the Third National Health and Nutrition Examination Survey. Public Health Nutr 4:781-786

5. Le Strat Y, Le Foll B (2011) Obesity and cannabis use: results from 2 representative national surveys. Am J Epidemiol 174:929-933

6. Rajavashisth TB, Shaheen M, Norris KC et al (2012) Decreased prevalence of diabetes in marijuana users: cross-sectional data from the National Health and Nutrition Examination Survey (NHANES) III. BMJ Open 2:e000494

7. Penner EA, Buettner H, Mittleman MA (2013) The impact of marijuana use on glucose, insulin, and insulin resistance among US adults. Am J Med 126:583-589

8. Alshaarawy O, Anthony JC (2015) Cannabis smoking and diabetes mellitus: results from meta-analysis with eight independent replication samples. Epidemiology 26:597-600

9. Friedman GD, Cutter GR, Donahue RP et al (1988) CARDIA: study design, recruitment, and some characteristics of the examined subjects. J Clin Epidemiol 41:1105-1116

10. Jacobs DR Jr, Hahn LP, Haskell WL, Pirie P, Sidney S (1989) Validity and reliability of a short physical activity history: CARDIA and the Minnesota Heart Health Program. J Cardiopulm Rehabil 9:448-459

11. Bild DE, Jacobs DR, Liu K et al (1996) Seven-year trends in plasma low-density-lipoprotein-cholesterol in young adults: the CARDIA Study. Ann Epidemiol 6:235-245

12. Lakoski SG, Herrington DM, Siscovick DM, Hulley SB (2006) C-reactive protein concentration and incident hypertension in young adults: the CARDIA study. Arch Intern Med $166: 345-349$
13. McDonald A, Van Horn L, Slattery M et al (1991) The CARDIA dietary history: development, implementation, and evaluation. J Am Diet Assoc 91:1104-1112

14. Sijtsma FP, Meyer KA, Steffen LM et al (2012) Longitudinal trends in diet and effects of sex, race, and education on dietary quality score change: the Coronary Artery Risk Development in Young Adults study. Am J Clin Nutr 95:580-586

15. Lockheart MS, Steffen LM, Rebnord HM et al (2007) Dietary patterns, food groups and myocardial infarction: a case-control study. Br J Nutr 98:380-387

16. Nettleton JA, Steffen LM, Ni H, Liu K, Jacobs DR Jr (2008) Dietary patterns and risk of incident type 2 diabetes in the Multi-Ethnic Study of Atherosclerosis (MESA). Diabetes Care 31:1777-1782

17. American Diabetes Association (2014) Diagnosis and classification of diabetes mellitus. Diabetes Care 37(Suppl 1):S81-S90

18. Muniyappa R, Sable S, Ouwerkerk R et al (2013) Metabolic effects of chronic cannabis smoking. Diabetes Care 36:2415-2422

19. Levendal RA, Schumann D, Donath M, Frost CL (2012) Cannabis exposure associated with weight reduction and beta-cell protection in an obese rat model. Phytomedicine 19:575-582

20. Weiss L, Zeira M, Reich S et al (2006) Cannabidiol lowers incidence of diabetes in non-obese diabetic mice. Autoimmunity 39: $143-151$

21. Nordstrom BR, Levin FR (2008) Substance abuse: cannabis-related disorders psychiatry. Wiley, Chichester, pp 1041-1057

22. Sarafian TA, Magallanes JA, Shau H, Tashkin D, Roth MD (1999) Oxidative stress produced by marijuana smoke. An adverse effect enhanced by cannabinoids. Am J Respir Cell Mol Biol 20:1286-1293

23. Kertesz SG, Khodneva Y, Richman J et al (2012) Trajectories of drug use and mortality outcomes among adults followed over 18 years. J Gen Intern Med 27:808-816

24. Morral AR, McCaffrey DF, Paddock SM (2002) Reassessing the marijuana gateway effect. Addiction 97:1493-1504

25. Harrison L, Martin S, Enev T, Harrington D (2007) Comparing drug testing and self-report of drug use among youths and young adults in the general population. Substance Abuse and Mental Health Services Administration, Office of Applied Studies, Rockville

26. Johnston L, O’Malley P, Bachman J, Schulenberg J, Miech R (2014) Monitoring the future national survey results on drug use, 1975-2013. Institute for Social Research, The University of Michigan, Ann Arbor 